\title{
De la prueba de esfuerzo a la predicción de muerte súbita cardiaca usando marcadores no invasivos
}

\author{
Cristina Pérez ${ }^{1}$, Esther Pueyo ${ }^{1,2}$, Juan Pablo Martínez ${ }^{1,2}$, Pablo Laguna ${ }^{1,2}$ \\ ${ }^{1}$ Afiliación: Biomedical Signal Interpretation and Computational Simulation (BSICoS) \\ Instituto de Investigación en Ingeniería de Aragón (I3A) \\ Universidad de Zaragoza, Mariano Esquillor s/n, 50018, Zaragoza, Spain. \\ Tel.+34-976762707, e-mail: cperez@unizar.es \\ ${ }^{2}$ Afiliación: CIBER-Bioingeniería, Biomateriales y Nanomedicina (CIBER BBN)
}

\section{Resumen}

En este trabajo se proponen marcadores no invasivos para estratificar pacientes según su riesgo de sufrir arritmias ventriculares que pueden desencadenar en muerte súbita cardiaca. Estos marcadores se basan en la respuesta del intervalo QT del ECG durante la prueba de esfuerzo, donde los cambios del ritmo cardiaco tienen un rango mayor de variación.

\section{Motivación}

La presencia de heterogeneidades electrofisiológicas en el miocardio ventricular da lugar a lo que se denomina dispersión de la repolarización ventricular. Las heterogeneidades pueden verse exacerbadas durante cambios bruscos de ritmo cardiaco (HR) cuando la velocidad a la que la repolarización se adapta a los cambios de ritmo varía de unas regiones ventriculares a otras. Esta mayor dispersión espaciotemporal puede desencadenar arritmias ventriculares y muerte súbita cardiaca (SCD) [1]. En los últimos años se han propuesto biomarcadores de riesgo arrítmico, entre ellos el tiempo de adaptación del intervalo QT a cambios abruptos en HR [2][3]. Sin embargo, contar con cambios bruscos en HR para medir el retardo no es sencillo en registros ambulatorios.

Asumiendo que la respuesta del QT a un cambio brusco de RR (inverso de HR) está gobernada por un sistema de primer orden, se sabe teóricamente que la constante de tiempo del sistema a una entrada rampa es la misma que a una entrada escalón (Fig. 1). Por tanto, evaluar el retardo frente a una entrada de HR
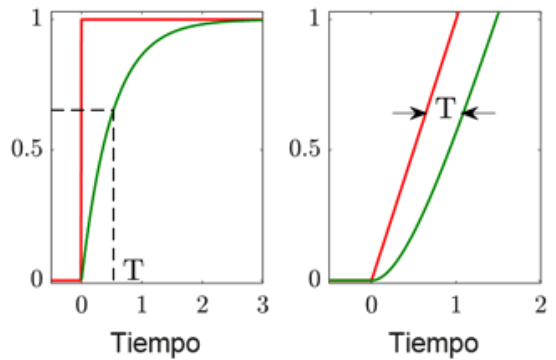

Figura 1. Respuestas teóricas de un sistema de primer orden cuando la entrada es un escalón (izq.) o una rampa (der.). tipo rampa debería aportar la misma información clínica que frente a un escalón. Por ello, y dado que en la prueba de esfurzo puede aproximarse la evolución temporal del HR como una rampa, se propone estudiar el retardo entre la rampa del HR y la del intervalo QT como marcador de riesgo arrítmico. Esto puede medirse tanto en la fase de ejercicio como de recuperación.

\section{Métodos}

\section{Datos y procesado de señal}

Se han evaluado 251 señales de ECG durante prueba de esfuerzo registradas en Tampere University Hospital (Finlandia). Según su historia clínica y los resultados de una angiografía coronaria, los pacientes se clasifican según su riesgo de padecer enfermedad de las arterias coronarias (CAD) en cuatro grupos: $\sin$ riesgo, riesgo bajo, medio y alto.

Sobre las 8 derivaciones estándar independientes, se aplica espacialmente un Análisis por Componentes Principales (PCA) para obtener la señal ECG correspondiente a la primera componente principal. Sobre ella se calculan las series de RR(i) y QT(i) aplicando un detector de picos, inicios y finales de ondas [4]. Los huecos que quedan en series debidos a intervalos que no ha sido posible medir se interpolan con splines, y se aplica un filtro basado en la mediana para eliminar artefactos. Por último, las series RR(i) y QT(i) se interpolan para conseguir las series temporales uniformemente muestreadas de $R R(n)$ y $Q T(n)$ con las que se trabaja.

\section{Estimación del tiempo de retardo}

En primer lugar se calcula la serie temporal de QT en el supuesto de que la respuesta entre el cambio de RR y de QT fuera instantánea $(\widehat{Q T}(n))$, es decir el QT que correspondería a cada $\mathrm{RR}$ en condiciones estacionarias. Esta serie $\widehat{Q T}(n)$ se calcula según un modelo hiperbólico estimado en tres ventanas estacionarias del registro, $\mathrm{W}_{1}, \mathrm{~W}_{2}$ y $\mathrm{W}_{3}$ en (Fig. 2a). En la Fig. 2b se muestran las series temporales $\widehat{Q T}(n)$ y $Q T(n)$ de un paciente. A continuación se

Revista “Jornada de Jóvenes Investigadores del I3A”, vol. 8 (Actas de la IX Jornada de Jóvenes Investigadores del I3A -11 de diciembre de 2020). ISSN 2341-4790. 
calcular el retardo $\tau^{*}$ que hace que el error entre las series $Q T(n)$ y $\widehat{Q T}\left(n-\tau^{*}\right)$ sea mínimo. Este retardo se calcula separadamente en la rampa de la fase de ejercicio, $\tau_{e}$, y en la rampa de la fase de recuperación, $\tau_{r}$, sugiriendo estos dos parámetros como marcadores de riesgo arrítmico. Se aplican técnicas de detección de puntos de cambio por medio de ajuste lineal para delimitar adecuadamente las dos rampas y estimar el retardo.

\section{Resultados y Conclusiones}

Los resultados obtenidos (Tabla 1) muestran que $\bar{\tau}_{e}$ aumenta según el grado de CAD, acorde a la hipótesis de que una adaptación más lenta está relacionada con el incremento de la dispersión ventricular y por tanto del riesgo cardiaco. Un comportamiento inverso se observa en los valores de $\bar{\tau}_{r}$. La diferencia entre estos dos retardos es mayor en los pacientes noCAD y de bajo riesgo que en los de riesgo medio $(p<0.01, p=0.01)$ y alto $(p<0.01$, $p<0.01$ ), sugiriendo $\Delta \tau=\tau_{r}-\tau_{e}$ como un marcador de riesgo más sensible. Los valores de retardo obtenidos son del mismo orden de magnitud que los reportados en [2], donde la constante de tiempo se estimó en zonas de cambio abrupto de HR en registros ambulatorios. Además, los resultados en pacientes noCAD siguen la misma tendencia que en [5], donde la adaptación del intervalo QT se mide ante cambios de tipo escalón de HR, tanto en aceleración como deceleración, en sujetos control.

Por último, hemos observado que las series $\widehat{Q T}(n)$ y $Q T(n)$ se superponen cuando se aproximan al pico de estrés en la fase de ejercicio (Fig. 2b). Esto lo relacionamos con los resultados del estudio [6] que muestra que el tiempo de adaptación de la repolarización ventricular a cambios de HR disminuye progresivamente conforme aumentan los niveles de estimulación simpática de los $\beta$-adrenoreceptores, como seguramente ocurre en

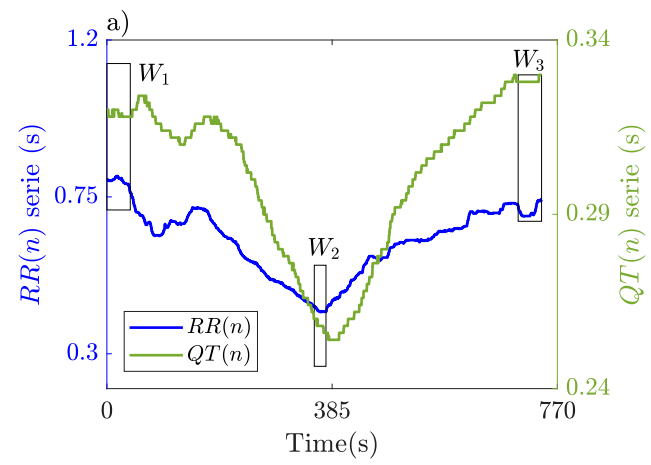

Tabla 1. Valor medio de los retardos en las rampas de ejercicio $\left(\bar{\tau}_{\mathrm{e}}\right)$ y recuperación $\left(\bar{\tau}_{\mathbf{r}}\right)$, y de su diferencia $\left(\bar{\Delta}_{\tau}\right)$.

\begin{tabular}{|c|c|c|c|c|}
\hline & noCAD & Bajo & Medio & Alto \\
\hline $\bar{\tau}_{\mathrm{e}}(\mathrm{s})$ & 20,96 & 17,33 & 39,13 & 35,68 \\
\hline$\overline{\boldsymbol{\tau}}_{\mathrm{r}}(\mathrm{s})$ & 49,05 & 46,22 & 37,73 & 38,07 \\
\hline$\overline{\boldsymbol{\Delta}}_{\tau}(\mathrm{s})$ & 28,09 & 28,89 & $-1,4$ & 2,39 \\
\hline
\end{tabular}

\section{Líneas Futuras}

- Evaluar estos marcadores para predecir SCD.

- Diseño de modelos biofísicos para evaluar la influencia del sistema nervioso autónomo en el tiempo de adaptación del intervalo QT.

\section{REFERENCIAS}

[1]. LAGUNA, P., CORTÉS, J.P., and PUEYO, E. Techniques for ventricular repolarization instability assessment from the ECG. Proceedings of the IEEE, 2016, vol. 104, no 2, p. 392-415.

[2]. PUEYO, E., et al. Characterization of QT interval adaptation to RR interval changes and its use as a riskstratifier of arrhythmic mortality in amiodaronetreated survivors of acute myocardial infarction. IEEE Transactions on biomedical engineering, 2004, vol. 51, no 9, p. 1511-1520.

[3]. PUEYO, E., et al. Mechanisms of ventricular rate adaptation as a predictor of arrhythmic risk. American Journal of Physiology-Heart and Circulatory Physiology, 2010, vol. 298, no 5, p. H1577-H1587.

[4]. MARTÍNEZ, J.P., et al. A wavelet-based ECG delineator: evaluation on standard databases. IEEE Transactions on biomedical engineering, 2004, vol. 51, no 4, p. 570-581.

[5]. PUEYO, E., MALIK, et al. A dynamic model to characterize beat-to-beat adaptation of repolarization to heart rate changes. Biomedical Signal Processing and Control, 2008, vol. 3, no 1, p. 29-43.

[6]. SAMPEDRO-PUENTE, DA., et al. Time course of low-frequency oscillatory behavior in human ventricular repolarization following enhanced sympathetic activity and relation to arrhythmogenesis. Frontiers in physiology, 2020, vol. 10, p. 1547.

Figura 2. a) Series RR(n) y QT(n) junto con las ventanas para estimar los parámetros del modelo. b) Ejemplo delimitación de las rampas de ejercicio y de recuperación, y sus retardos obtenidos comparando $Q T(n)$ y $\widehat{Q T}\left(n-\tau^{*}\right)$.

la ultima fase de la pruecba de esfuerzo donde se observa este acercamiento. 